\title{
Correction to: Relatively low and moderate pre-fracture serum 25-hydroxyvitamin D levels associated with the highest survival in elderly hip fracture patients in Finland: a minimum 3-year follow-up
}

\author{
I. Nurmi-Lüthje ${ }^{1}$ (D) R. Tiihonen ${ }^{2} \cdot$ E-L. Paattiniemi ${ }^{3} \cdot$ H. Sarkkinen ${ }^{3} \cdot$ H. Naboulsi ${ }^{4} \cdot$ S. Pigg ${ }^{5} \cdot$ J-P. Kaukonen $^{6}$. \\ M. Kataja ${ }^{7}$ P. Lüthje
}

Published online: 3 December 2021

(C) International Osteoporosis Foundation and National Osteoporosis Foundation 2021

\section{Correction to: Osteoporosis International https://doi.org/10.1007/s00198-021-06094-z}

In Table 2 of this article, the last column heading 'B/ $(\mathrm{A}+\mathrm{B}+\mathrm{C})^{\prime}$ should have read $\mathrm{B} /(\mathrm{A}+\mathrm{C}+\mathrm{D})$ '.

The original article has been corrected.

Publisher's note Springer Nature remains neutral with regard to jurisdictional claims in published maps and institutional affiliations.

The original article can be found online at https://doi.org/10.1007/ s00198-021-06094-z.

I. Nurmi-Lüthje

ilona.nurmi@pp.inet.fi

1 Department of Public Health, Helsinki University, Mannerheimintie 172, FI-00300 Helsinki, Finland

2 Department of Orthopedics and Traumatology, Päijät-Häme Central Hospital, Keskussairaalankatu 7, FI-15850 Lahti, Finland

3 Centre for Laboratory Services, Päijät-Häme Social and Health Care Group, Keskussairaalankatu 7, FI-15850 Lahti, Finland

4 Joint Authority for Päijät-Häme Social and Health Care Services, Päijät-Häme Social and Health Care Group, Keskussairaalankatu 7, FI-15850 Lahti, Finland

5 Kouvola Health Center, Marjoniementie 10, FI-45100 Kouvola, Finland

6 Terveystalo Lahti, Aleksanterinkatu 11-13, FI-15110 Lahti, Finland

7 National Institute for Health and Welfare, Mannerheimintie 166, FI-0027 Helsinki, Finland

8 North Kymi Hospital, FI-45750 Kuusankoski, Finland 
Table 2 Cumulative mortality of 244 patients at 12, 24 and 36 months according to serum 25-hydroxyvitamin D level $(\mathrm{nmol} / \mathrm{L})$

\begin{tabular}{|c|c|c|c|c|c|c|c|c|c|c|c|}
\hline \multirow[b]{3}{*}{ Months } & \multirow{2}{*}{\multicolumn{2}{|c|}{$\begin{array}{l}\text { Group A } \\
<50 \mathrm{nmol} / \mathrm{L} \\
(n=55)\end{array}$}} & \multirow{2}{*}{\multicolumn{2}{|c|}{$\begin{array}{l}\text { Group B } \\
\begin{array}{l}50-74 \mathrm{nmol} / \mathrm{L} \\
(n=75)\end{array}\end{array}$}} & \multirow{2}{*}{\multicolumn{2}{|c|}{$\begin{array}{l}\text { Group C } \\
\begin{array}{l}75-99 \mathrm{nmol} / \mathrm{L} \\
(n=69)\end{array}\end{array}$}} & \multirow{2}{*}{\multicolumn{2}{|c|}{$\begin{array}{l}\text { Group D } \\
\begin{array}{l}\geq 100 \mathrm{nmol} / \mathrm{L} \\
(n=45)\end{array}\end{array}$}} & \multicolumn{2}{|c|}{$\begin{array}{l}\text { Total } \\
\left(n=244^{\mathrm{a}}\right)\end{array}$} & \multirow{3}{*}{$\begin{array}{l}\mathrm{B} /(\mathrm{A}+\mathrm{C}+\mathrm{D}) \\
\mathrm{p}^{\mathrm{b}}\end{array}$} \\
\hline & & & & & & & & & \multirow[b]{2}{*}{$n$} & \multirow[b]{2}{*}{$\%$} & \\
\hline & $n$ & $\%$ & $n$ & $\%$ & $n$ & $\%$ & $n$ & $\%$ & & & \\
\hline 12 & 15 & 27.3 & 8 & 10.7 & 12 & 17.4 & 12 & 26.7 & 47 & 19.3 & 0.0155 \\
\hline 24 & 21 & 38.2 & 12 & 16.0 & 16 & 23.2 & 14 & 31.1 & 63 & 25.8 & 0.0130 \\
\hline 36 & 24 & 43.6 & 20 & 26.7 & 24 & 34.8 & 18 & 40.0 & 86 & 35.2 & 0.0412 \\
\hline
\end{tabular}

Kruskal-Wallis 1.809 , df 3, n.s.; ${ }^{\mathrm{a}}$ one sample missing; ${ }^{\mathrm{b}}$ Fisher's exact test 